\title{
Evaluation and optimization of multilayer designs for astronomical x-ray telescopes using a field-of-view- and energy-dependent figure of merit
}

\author{
Peter H. Mao ${ }^{a}$, Leon Bellan ${ }^{a}$, Fiona A. Harrison ${ }^{a}$, David L. Windt ${ }^{b}$, Finn E. Christensen ${ }^{c}$ \\ ${ }^{a}$ California Institute of Technology, MC 220-47, Pasadena, CA 91125 \\ ${ }^{b}$ Columbia Astrophysics Laboratory, Columbia University \\ 550 W. 120th St., New York, NY 10027 \\ ${ }^{c}$ Danish Space Research Institute, Juliane Maries Vej 30 \\ Copenhagen, DK-2100
}

\begin{abstract}
The three most important quantities used to assess the performance of astronomical x-ray telescope optics are the on-axis collecting area, the field of view, and the half-power diameter. The first two quantities depend on the mirror packing arrangement and the multilayer coating design. In order to optimize the coating design, we have developed a figure-of-merit (FOM) that accounts for the coating response over a specified range of energies and off-axis angles. We present an example where we have used this FOM to optimize a specific coating design for the High Energy Focusing Telescope (HEFT) and to understand tradeoffs between performance and coating thickness.
\end{abstract}

Keywords: multilayer optimization,x-ray optics

\section{INTRODUCTION}

Focusing $\mathrm{x}$-rays at energies greater than $10-15 \mathrm{keV}$ becomes impractical with grazing incidence metallic mirrors, such as those used on Chandra and XMM, because the critical angle for total external reflection, above which x-rays are absorbed rather than reflected, is roughly proportional to $1 / E$, where $E$ is the photon energy. The relation between critical angle and photon energy means that the field of view and the ratio of mirror diameter to focal length of telescopes using single layer metallic mirrors also scale as $1 / E$. Such telescopes would then necessarily have small fields of view and either proportionally smaller diameter optics or longer focal lengths. Thin film multilayer coatings, using alternating layers of high and low atomic number $(\mathrm{Z})$ materials, are the leading technology for extending $\mathrm{x}$-ray focusing to higher energies. The physics of the coatings, with regard to reflection of x-rays, is similar to that of Bragg crystals. The advantage of multilayer coatings over Bragg crystals is that we can design the coatings for broadband reflectivity by depth grading the bilayer thicknesses in the coating, effectively varying the "lattice constant" of the coating in the direction normal to the surface.

The current literature on multilayer optimization almost exclusively deals with maximizing integrated reflectance ${ }^{1,2}$ or matching the reflectance to a desired function ${ }^{1,3,4}$ either over a range of photon energies at a single reflection angle or over a range or reflection angles at a single photon energy. Optimization methods that optimize a multilayer design for a single angle or a single photon energy may be useful for laboratory applications where reflection angles and/or photon energies are fixed. For a general-purpose astronomical hard x-ray telescope, however, maximizing the effective area over a given energy range and field of view (i.e. a relatively wide range of incidence angles) is more important than producing a specific response at a single energy or reflection angle. For example, galaxy clusters and nearby radio galaxies are extended at the few arcminute level. In addition, for a balloon-borne instrument, one must account for instabilities in the pointing of the telescope which can also be at the few arcminute level. For these reasons, the off-axis performance of astronomical x-ray telescopes deserves at least as much attention as the often-quoted on-axis performance.

To this end, we devised a figure of merit function that is the field-of-view- and energy-weighted average effective area of a telescope's optics. ${ }^{5}$ The calculation of the figure of merit requires specification of the geometry of the

Further author information: (Send correspondence to P.H.M.)

P.H.M.: E-mail: peterm@srl.caltech.edu 
telescope optics, weighting functions for spectral and angular response and the matrix of multilayer reflectivity vs. energy and incidence angle. Since the publication of Mao et al, 1999 (henceforth M99), we have refined the calculation of the angular weighting function and incorporated an iterative search routine into the multilayer optimization program.

In the next section, we discuss the geometry of and the reflection incidence angle distributions on a conicalapproximation Wolter I telescope. We then discuss various methods for calculating the effective area of a telescope in Section 3, followed by a derivation of the figure of merit function in Section 4. In Section 5, we present an optimization using Joensen's parameterization for a graded multilayer.

\section{GEOMETRY OF THE OPTICS}

The first step in multilayer optimization is specification of the geometry of the telescope's optics. Off-axis performance is strongly affected by vignetting, so the optimum geometry is determined by balancing on-axis collecting area with off-axis vignetting. The geometry is also crucial to the multilayer design optimization because the reflection angle distribution on each mirror is needed to calculate the throughput of the optics. For both tasks, we use a Monte Carlo ray trace program with roughly $10^{8}$ input events. The off-axis angle distribution used in the ray trace affects the optimization of the geometry of the optics and determines the reflection angle distributions that will be used to calculate the figure of merit. We use a uniform distribution of off-axis angles between 0 and $3 \mathrm{mrad}$, with the largest angle set by the size of our detectors and the focal length of the telescope. To design geometries and multilayers with even greater off-axis performance (at the expense of on-axis performance), one would use an input distribution that favors off-axis photons.

The figure of merit and optimization technique that we developed is part of the High Energy Focusing Telescope (HEFT) project, ${ }^{6}$ a balloon-borne telescope using thermally formed glass substrates for the mirrors. ${ }^{7}$ The use of glass substrate forces us to use a conical approximation to the Wolter I (parabola/hyperbola) geometry. Because the typical reflection angles are so small (1-5 mrad), using the conical approximation only incurs roughly $1^{\prime}$ distortion in the image of a point source at the edge of HEFT's field of view ( $3 \mathrm{mrad}$ off-axis). The HEFT substrates are 0.3 $\mathrm{mm}$ thick, $20 \mathrm{~cm}$ long sheets of Schott DESAG D263 glass. A schematic of the optics' geometry and the relevant angles is shown in Figure 1. The half-opening angles of the mirror shells are set by the following equations:

$$
\begin{gathered}
\alpha_{i}=r_{i} /(4 f) \\
\beta_{i}=3 \alpha_{i}
\end{gathered}
$$

where $\alpha_{i}$ and $\beta_{i}$ are the respective half-opening angles of the primary and secondary shells, $r_{i}$ is the radius of the $i$ th shell at the plane between the primary and secondary mirror sections ( $4-12 \mathrm{~cm}$ for HEFT), and $f$ is the focal length of the telescope ( $6 \mathrm{~m}$ for HEFT).

The difference in radii between consecutive concentric mirror shells produces a tradeoff between on- and off-axis collecting areas. On-axis collecting area is maximized when the inner radius of the $i+1$ st primary shell lies on the same coaxial cylinder as the outer edge of the $i$ th primary shell. Increasing the radial gap (cf Figure 1) between consecutive shells improves off-axis collecting area at the expense of on- and nearly on-axis area. We explored two methods of defining the extra gap between mirror shells: a constant gap between all shells, such that the difference in radii between consecutive shells is

$$
\Delta r_{i, i+1}=\alpha_{i} l+\text { const. }
$$

where $l$ is the length of the mirror along the optical axis, and a radius dependent gap where the gap between the consecutive shells is

$$
\Delta r_{i, i+1}=\alpha_{i} l+\xi \alpha_{i} l
$$

where $\xi$ is the variable gap parameter. When $\xi=0$, there is no additional gap between shells; when $\xi=1$, the gap is equal to the projected radial width of the primary shell. From ray tracing with perfect reflectivity, $R=1$, we determined that the angularly averaged collecting area (the fraction of collected events multiplied by the illuminated area) is maximized with a constant gap of $0.17 \mathrm{~mm}$ between consecutive shells (see Figure 2). A variable gap with $\xi=0.26$ maximizes the area for that method, but falls short of the constant gap geometry by a fraction of a percent. 


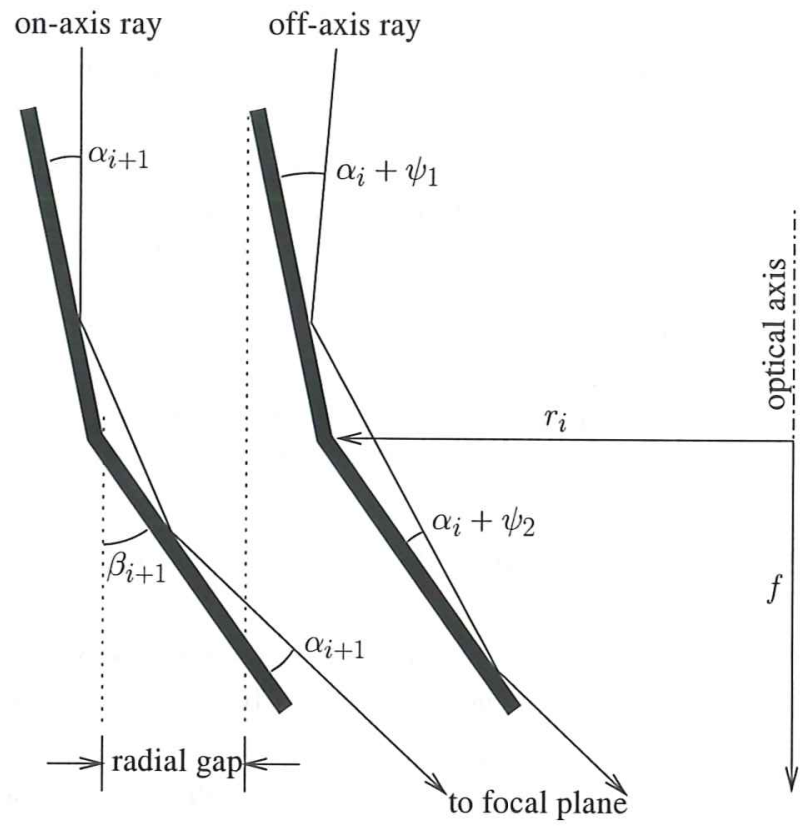

Figure 1. Geometry of conical-approximation Wolter I optics with primary and secondary reflection angles for onand off-axis rays.

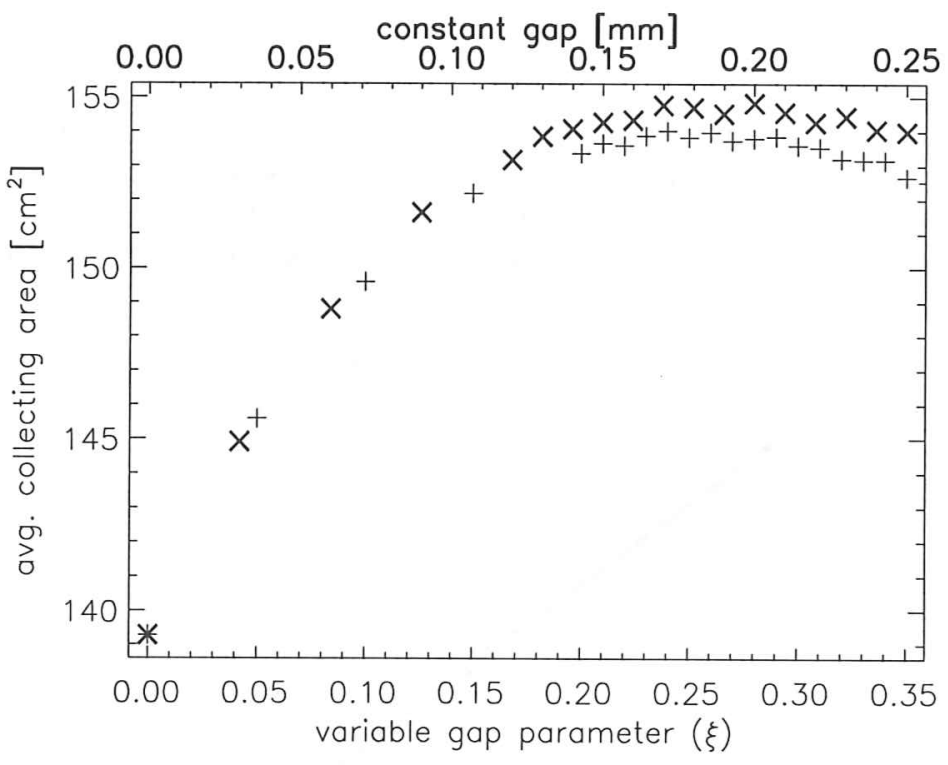

Figure 2. Angularly averaged collecting area vs radial gap between mirror shells. Variable gap results $(+)$ are plotted against the bottom scale and constant gap results $(x)$ are plotted against the top scale. Each area is determined by ray tracing $10^{8}$ events uniformly distributed in off-axis angles between 0 and $3 \mathrm{mrad}$, and uniformly distributed spatially over the $12 \mathrm{~cm}$ radius aperture. The standard deviation in the estimate of the area is 0.025 $\mathrm{cm}^{2}$.

\section{EFFECTIVE AREAS}

The effective area of a telescope is the product of the collecting area and the reflectivities of the mirrors. On-axis effective area is the easiest area to calculate, since the incidence angles on the primary and secondary mirrors are identical. The area of the $i$ th shell is thus

$$
A_{i}(E)=\left(2 \pi r_{i} \alpha_{i} l\right) \cdot\left(\left[R\left(E, \alpha_{i}\right)\right]^{2}\right),
$$


where $l$ is the length of the mirror along the optical axis and $E$ is the energy of the photon. The first term in the above equation is the projected area of the primary mirror, and the second term gives the reflection efficiency.

For off-axis photons, the incidence angles on the two mirrors differ. To calculate the off-axis effective area we need the incidence angle distribution on each mirror, whose magnitude is normalized to the collecting area. Consider photons arriving from a source at off-axis angle $\psi$. The incidence angles on the primary and secondary mirrors are $\theta_{1}=\alpha+\psi_{1}$ and $\theta_{2}=\alpha+\psi_{2}$, respectively. The angles $\psi_{1}$ and $\psi_{2}$ have values between $-\psi$ and $\psi$, depending on the difference between the azimuthal angle of the source and the azimuthal position of the point of reflection, $\Delta \phi$. For example, when $\Delta \phi=0, \psi_{1}=-\psi$ and when $\Delta \phi=\pi, \psi_{1}=\psi$. In M99, we made the approximation that $\psi_{1}=-\psi_{2}$. This approximation allowed us calculate the effective area using only the incidence angle distribution on the primary mirrors. The 1-dimensional function $W_{\text {inc }}\left(\alpha_{i}, \psi_{1}\right)$ is the incidence angle distribution generated by the ray trace with off-axis angles uniformly distributed between 0 and $3 \mathrm{mrad}$, and the effective area is

$$
A_{i}(E)=\int_{-\psi}^{\psi} \mathrm{d} \psi_{1} W_{\mathrm{inc}}\left(\alpha_{i}, \psi_{1}\right) \cdot\left[R\left(E, \alpha_{i}+\psi_{1}\right) R\left(E, \alpha_{i}-\psi_{1}\right)\right]
$$

where $\psi$ is the half angle of the full field of view. There is no explicit integration in the azimuthal direction in Equation 6 because it is already incorporated into the distribution function by the ray trace.

Here we eliminate the approximation $\psi_{1}=-\psi_{2}$ used in M99 by explicitly keeping track of the correlation between $\psi_{1}$ and $\psi_{2}$ in the ray trace and generating a 2-dimensional incidence angle distribution for each set of mirror shells. A contour plot of the 2-dimensional incidence angle distribution, $W_{\text {inc }}\left(\alpha_{i}, \psi_{1}, \psi_{2}\right)$, and the projection of the distribution onto the $\psi_{1}$-axis, $W_{\text {inc }}\left(\alpha_{i}, \psi_{1}\right)$, are shown in Figure 3. With the 2-D distribution, the effective area is

$$
A_{i}(E)=\int_{-\psi}^{\psi} \mathrm{d} \psi_{1} \int_{-\psi}^{\psi} \mathrm{d} \psi_{2} W_{\text {inc }}\left(\alpha_{i}, \psi_{1}, \psi_{2}\right) \cdot\left[R\left(E, \alpha_{i}+\psi_{1}\right) R\left(E, \alpha_{i}+\psi_{2}\right)\right] .
$$

For our purposes, calculating the effective area from Equation 6 is nearly as good as calculating the area from Equation 7. Using the exact formula for the area, however, allows us to apply this technique to other reflection geometries such as Kirkpatrick-Baez or a true parabola/hyperbola Wolter I. In addition, extending the technique to even more reflections is trivial: we simply add one dimension to the incidence angle distribution matrix for each additional reflection.

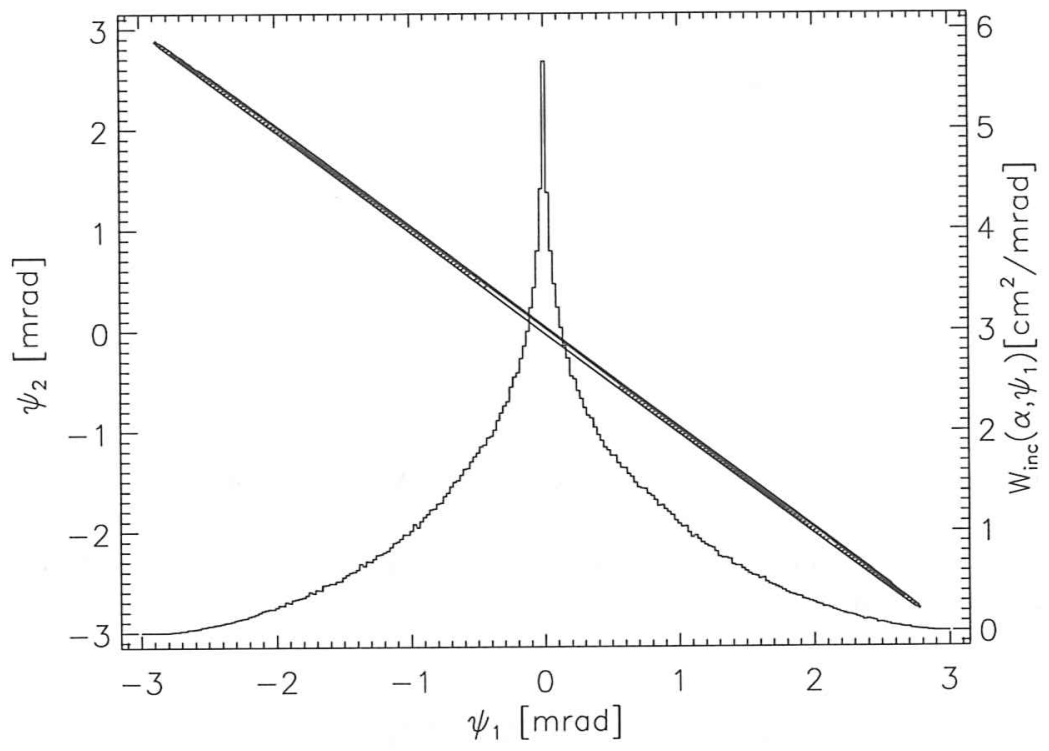

Figure 3. The 2-dimensional reflection angle distribution, $W_{\text {inc }}\left(\alpha=5 \mathrm{mrad}, \psi_{1}, \psi_{2}\right)$, and its projection onto the $\psi_{1}$-axis, $W_{\text {inc }}\left(\alpha=5 \mathrm{mrad}, \psi_{1}\right)$. The distribution shown here is for the outermost set of shells of the HEFT design. 


\section{FIGURE OF MERIT FUNCTION}

We use the angularly weighted effective area to calculate the figure of merit (FOM) for specific multilayer bilayer distributions. In the FOM we include an additional, energy-dependent weighting function, $W_{E}(E)$, that allows flexibility in defining the spectral response of an optimized design. We use an energy weighting function that increases with energy because almost all astronomical sources have falling $\mathrm{x}$-ray spectra. The energy weighting function is normalized so that its integral over the energy range of interest is unity. The FOM is thus the weighted energy integral of the field-of-view averaged effective area for each mirror shell, summed over all mirrors:

$$
\mathrm{FOM}=\frac{\sum_{i=1}^{N} \int_{E_{\min }}^{E_{\max }} \mathrm{d} E A_{i}(E) W_{E}(E)}{E_{\max }-E_{\min }} .
$$

The advantages of using Equation 8 as a figure of merit are: (1) it takes into account the performance across the field of view, (2) it can be used to compare different telescope designs (given the same weighting functions), and (3) it allows us to automate the optimization of multilayer designs for a given optics geometry.

\section{OPTIMIZATION EXAMPLE}

We determine the optimized multilayer design by finding the multilayer bilayer distribution that maximizes the figure of merit (Equation 8). This approach is CPU-intensive, however, as each time the multilayer parameters are changed, the entire reflectance matrix has to be recalculated, and the calculation of the reflectance matrix is the most time consuming part of the optimization. For example, a 333-MHz Sun Enterprise 3000 server takes 6 minutes to calculate a $160 \times 500$ reflectance matrix for a multilayer containing 250 bilayers. We reduce the time to optimize a design by using parallel processing to calculate the reflectance matrices. We use the amoeba algorithm from Numerical Recipes, which is based on the downhill simplex method of Nelder and Mead, ${ }^{8}$ to adjust parameters and maximize the figure of merit.

As an example of multilayer design optimization, we present here the optimization of a subset of the HEFT optics using Joensen's parameterization for x-ray multilayers ${ }^{9}$ assuming W/Si multilayers. For HEFT, we split the mirrors into ten groups of shells, divided logarithmically in radius, and optimize Joensen's multilayer parameterization individually for each group of shells. We do not use a single design for all mirrors because the incidence angle distribution of each shell is centered the half-opening angle of its primary shell, and the half-opening angles of the inner and outermost shells differ by a factor of three. Complete information on full optimization of the HEFT design is given in M99.

Joensen's multilayer parameterization is a power law distribution of $N$ bilayers with three adjustable constants:

$$
d(i)=a /(b+i)^{c} .
$$

The index $i$ labels the bilayers from 1 to $N$ where the $N$ th bilayer is adjacent to the substrate. The bilayer thickness distribution can be specified with the four parameters $a, b, c$ and $N$. It is, however, more convenient to specify $d_{\min }=d(N), d_{\max }=d(1), c$, and $N$ because Bragg's formula can be used to determine the necessary range in bilayer thicknesses. Since we use Joensen's parameterization only for the bilayer thickness distribution, we need also to specify the fractional thickness of the high Z layer within each bilayer $\left(\Gamma_{i}\right)$. A Joensen-parameterized graded multilayer is thus specified with $N+4$ parameters. We further reduce the number of adjustable parameters by restricting designs to those with a single value of $\Gamma$, cutting the number of parameters from $N+4$ to just five. Finally, we use Bragg's equation to specify $d_{\min }$ and $d_{\max }$ (see M99), leaving $N, c$ and $\Gamma$ as the optimization parameters.

With $d_{\min }$ and $d_{\max }$ constrained, the thickness of the multilayer coating is largely determined by the number of bilayers, and only weakly affected by $c$ and $\Gamma$. In order to obtain the information needed to make the tradeoff between coating thickness (and thus fabrication time) and performance, we step through a range of values of $N$, using amoeba only to optimize $c$ and $\Gamma$ at each value of $N$. We typically start with a small value of $N$ that we know from experience is less than optimal (usually 100-150) and increment $N$ by a factor of 1.25 after each optimization on $c$ and $\Gamma$. The process is repeated until the optimum figure of merit for successive values of $N$ decreases. Figure 4 shows the results of an optimization starting with $N=150$ on HEFT's $\alpha=2.32-2.59$ mrad mirror shells. The design optimizes at around $N=363$. Although $1.27 \mu \mathrm{m}$ is a reasonable coating thickness, we can reduce the coating thickness by more than $30 \%$ by using the $N=233$ design, which has roughly $98 \%$ of the area of the optimum design. 


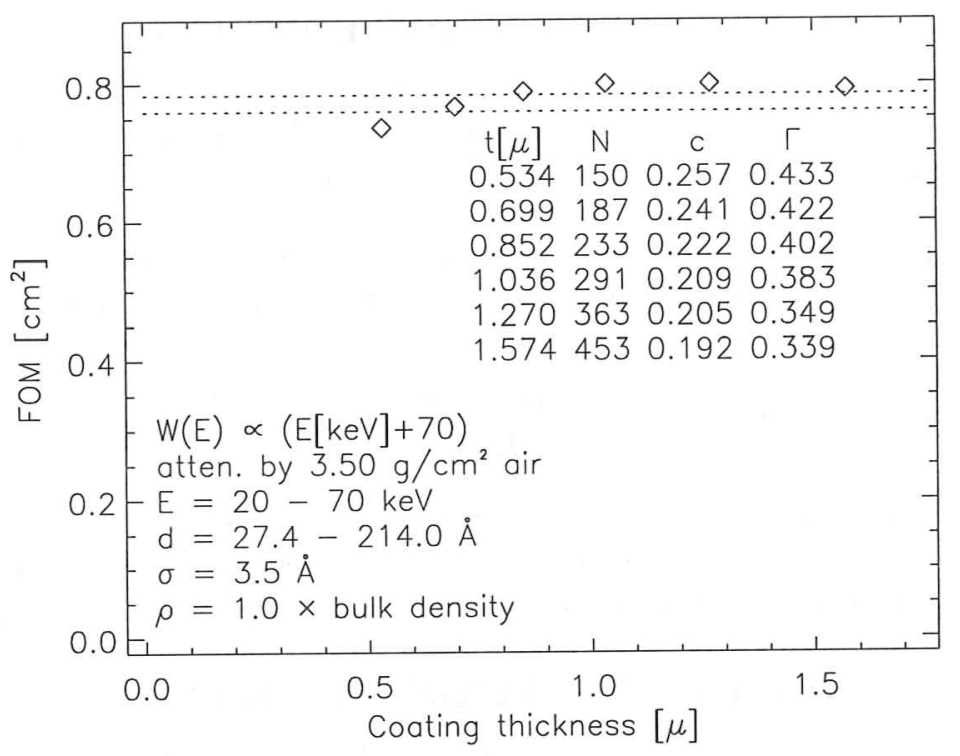

Figure 4. Figure of merit vs. coating thickness for HEFT's $\alpha=2.32-2.59$ mrad mirrors. The dashed lines indicate levels of $98 \%$ and $95 \%$ of the optimum figure of merit. $\sigma$ is the RMS interface width.

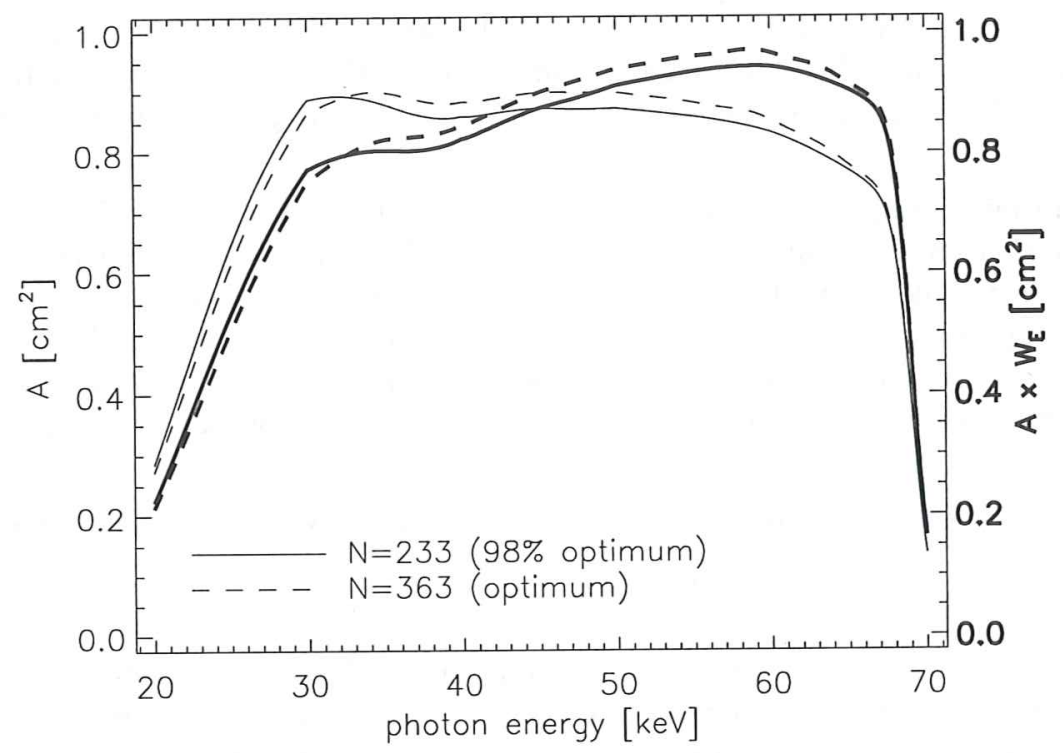

Figure 5. Angularly averaged effective area and energy weighted, angularly averaged effective area (in bold). $W_{E} \propto E[\mathrm{keV}+70]$. The solid line, $N=233$, corresponds to the HEFT design with a figure of merit that is $98 \%$ of optimum. The dashed line, $N=363$, is our best estimate of optimum design. The figure of merit that we calculate is the average value of the energy weighted, angularly averaged effective area.

The angularly averaged spectral response of the optimized and "98\% optimized" designs for this subset of HEFT's shells is shown in Figure 5 both with and without the energy weighting function, $W_{E} \propto E[\mathrm{keV}]+70$, used to calculate the figure of merit. The spectral response to on-axis point sources and point sources at three off-axis angles is shown in Figure 6. The large in on-axis response around $35 \mathrm{keV}$ is not of concern to us because that feature is averaged out by the other mirror shells. Figure 7 shows the effective area of the full HEFT design for an on-axis source and for off-axis sources at three angles. The full width at half-maximum of the effective area for HEFT is $3.0 \mathrm{mrad}$ at energies less than $45 \mathrm{keV}$ and gradually decreases to $2.0 \mathrm{mrad}$ at $70 \mathrm{keV}$. 


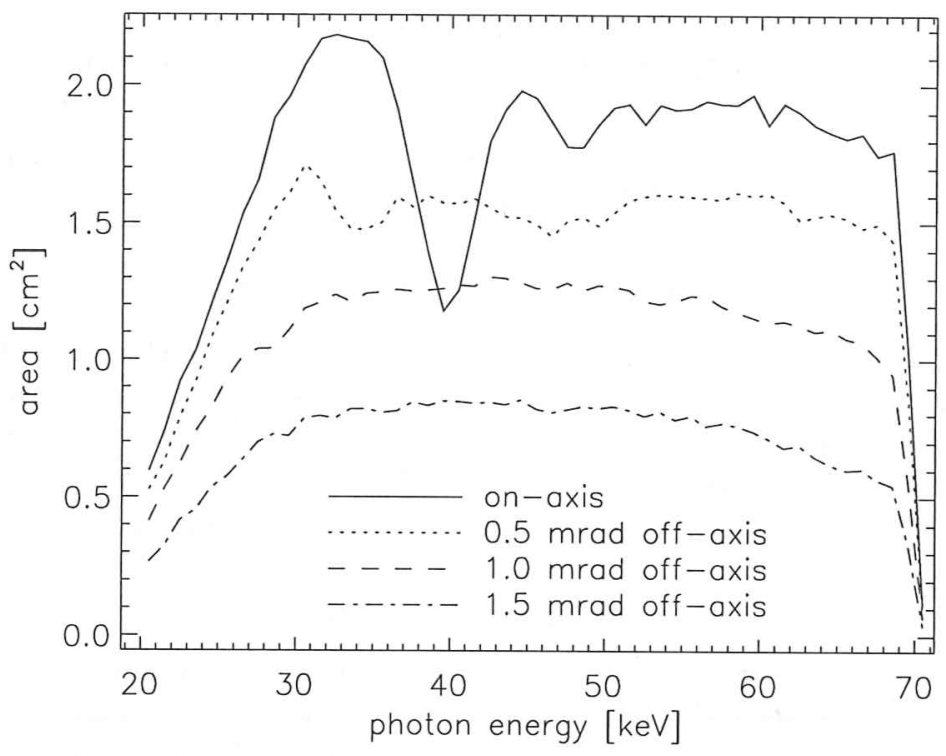

Figure 6. On- and off-axis effective area vs photon energy for mirror shells with $\alpha$ between 2.32 and 2.59 mrad.

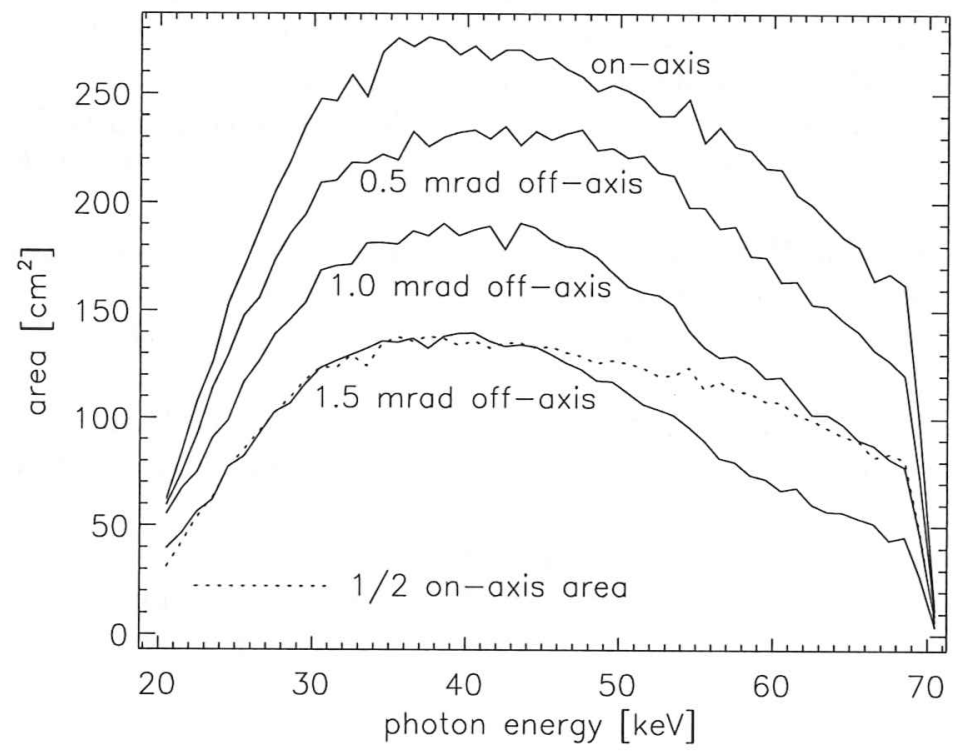

Figure 7. Effective area of the full 14-module HEFT design for on-axis sources and off-axis point sources at 0.5 , 1.0 , and $1.5 \mathrm{mrad}$ off-axis.

\section{CONCLUSION}

We have developed a technique for evaluating and optimizing multilayer designs for X-ray telescopes that accounts for performance over the field of view and the spectral range of the instrument. The technique gives a more comprehensive evaluation of telescope and multilayer design than a calculation of the on-axis effective area alone does.

The technique starts with Monte-Carlo ray tracing to optimize the geometry of the optics and to calculate the reflection angle distributions, given an input off-axis angle distribution. We calculate the effective area from the incidence angle distributions and the multilayer's reflectance matrix using either a function that is specific to the Wolter 1 geometry and uses the approximation $\psi_{1}=-\psi_{2}$ (Equation 6), or a function that can be used for any 2-reflection optical system and is as exact as the Monte Carlo generated incidence angle distributions allow it to be (Equation 7). 
Once we compute the effective area, we calculate a figure of merit (Equation 8) that folds in the spectral response with an energy weighting function. In the optimization example, we use Joensen's parameterization for depth graded x-ray multilayers; the same methods can be extended, in principle, to other multilayer designs.

In addition to multilayer designs optimization, our figure of merit can also be used to make objective comparisons of x-ray telescopes with different geometries and multilayer designs.

\section{ACKNOWLEDGMENTS}

This research was supported in part by NASA Supporting Research and Technology (SR\&T) grant NAG5-5128, NASA Explorer Technology grant NAG5-8535, and by a NASA Graduate Student Researchers Program (GSRP) Fellowship (P.H. Mao). This research was performed in part using the Center for Advanced Computing Resources (CACR) parallel computer system operated by Caltech. P.H. Mao thanks Prof. Thomas Prince for access to CACR's services and Dr. Stuart Anderson for advice and guidance on parallel computing codes.

\section{REFERENCES}

1. A. G. Michette and Z. Wang, "Optimisation of depth-graded multilayer coatings for broadband reflectivity in the soft X-ray and EUV regions," Optics Communications 177, pp. 47-55, Apr. 2000.

2. K. Yamashita, H. Kunieda, Y. Tawara, K. Tamura, Y. Ogasaka, K. Haga, T. Okajima, Y. Hidaka, S. Ichimaru, S. Takahashi, A. Goto, H. Kito, Y. Tsusaka, K. Yokoyama, and S. Takeda, "New design concept of multilayer supermirrors for hard x-ray optics," Proc. SPIE 3766, pp. 327-335, Sept. 1999.

3. I. V. Kozhevnikov, I. N. Bukreeva, and E. Ziegler, "Theoretical study of multilayer x-ray mirrors with a wide spectral band of reflection," Proc. SPIE 3448, pp. 322-331, Dec. 1998.

4. V. V. Protopopov, A. V. Tikhonravov, A. V. Voronov, M. K. Trubetskov, and G. W. Debell, "Optimal design of graded x-ray multilayer mirrors in the angular and spectral domains," Proc. SPIE 3766, pp. 320-326, Sept. 1999.

5. P. H. Mao, F. A. Harrison, D. L. Windt, and F. E. Christensen, "Optimization of graded multilayer designs for astronomical x-ray telescopes," Appl. Opt. 38, pp. 4766-4775, Aug. 1999.

6. F. A. Harrison et al., "Development of the High-Energy Focusing Telescope (HEFT) balloon experiment," Proc. SPIE 4012, Mar. 2000.

7. C. J. Hailey, S. Abdali, F. E. Christensen, W. W. Craig, T. R. Decker, F. A. Harrison, and M. Jimenez-Garate, "Substrates and mounting techniques for the High-Energy Focusing Telescope (HEFT)," Proc. SPIE 3114, pp. 535-543, Oct. 1997.

8. J. A. Nelder and R. Mead Computer Journal 7, pp. 308-313, 1965.

9. K. Joensen, P. Voutov, A. Szentgyorgyi, J. Roll, P. Gorenstein, P. Hoghoj, and F. Christensen, "Design of grazing-incidence multilayer supermirrors for hard-x-ray reflectors," Appl. Opt. 34(34), pp. 7935-7944, 1995. 